\title{
Immediate screening method for predicting the necessity of massive transfusions in trauma patients: a retrospective single-center study
}

Tetsuya Yumoto ${ }^{*}$, Atsuyoshi lida, Takahiro Hirayama, Kohei Tsukahara, Naoki Shiba, Hideo Yamanouchi, Keiji Sato, Toyomu Ugawa, Shingo Ichiba and Yoshihito Ujike

\begin{abstract}
Background: Hemostatic resuscitation might improve the survival of severely injured trauma patients. Our objective was to establish a simplified screening system for determining the necessity of massive transfusions (MT) at an early stage in trauma cases.

Methods: We retrospectively analyzed the cases of trauma patients who had been transported to our institution between November 2011 and October 2013. Patients who were younger than 18 years of age or who were confirmed to have suffered a cardiac arrest at the scene or on arrival were excluded. MT were defined as transfusions involving the delivery of $\geq 10$ units of red blood cell concentrate within the first $24 \mathrm{~h}$ after arrival.

Results: A total of 259 trauma patients were included in this study (males: 178, 69\%). Their mean age was $49 \pm 20$, and their median injury severity score was 14.4 . Thirty-three (13\%) of the patients required MT. The presence of a shock index of $\geq 1$, a base excess of $\leq-3 \mathrm{mmol} / \mathrm{L}$, or a positive focused assessment of sonography for trauma (FAST) result was found to exhibit sensitivity and specificity values of 0.97 and 0.81 , respectively, for predicting the necessity of MT. Furthermore, this method displayed an area under the receiver operating characteristic curve of 0.934 (95\% confidence interval, 0.891-0.978), which indicated that it was highly accurate.
\end{abstract}

Conclusions: Our screening method based on the shock index, base excess, and FAST result is a simple and useful way of predicting the necessity of MT early after trauma.

Keywords: Massive transfusion, Shock index, Base excess, Focused assessment of sonography for trauma

\section{Background}

Uncontrolled hemorrhaging is a major cause of death in trauma patients [1]. In addition to undergoing surgical intervention or angiographic embolization to control the bleeding, patients who suffer such hemorrhaging can also require massive transfusions (MT). Damage control resuscitation (DCR), which integrates permissive hypotension, hemostatic resuscitation, and damage control surgery is a crucial strategy for treating severely injured trauma patients [2,3]. Damage control-based surgery focuses on controlling bleeding and contamination, and hemostatic resuscitation aims to manage coagulopathy as soon as

\footnotetext{
* Correspondence: tyumoto@cc.okayama-u.ac.jp

Advanced Emergency and Critical Care Medical Center, Okayama University Hospital, 2-5-1 Kita-ku, Shikata-cho, Okayama-shi, Okayama 700-8558, Japan
}

possible via the early induction of MT protocols involving a balanced ratio of blood products and restrictive fluid replacement to prevent the development of coagulopathy $[4,5]$. Although several models for predicting the necessity of MT have been reported [6-9], they are too complicated for practical use. The aim of this study is to establish a simple screening method for predicting the necessity of MT at a very early stage in trauma cases.

\section{Methods \\ Study population \\ Data for traumatically injured patients who were trans- ported to Okayama University Hospital between November 1, 2011 and October 31, 2013 were retrospectively collected. Patients who were younger than 18 years of age or were confirmed to have suffered a cardiac arrest at the}


scene or on arrival at the emergency department (ED) were excluded. This study was approved by the institutional review board at the Okayama University.

\section{Definition of massive transfusions}

MT were defined as transfusions of $\geq 10$ units of red blood cell concentrate (RCC) that were administered within the first $24 \mathrm{~h}$ after the patient's arrival at hospital. Patients who were bleeding and were expected to require high-volume transfusions within $24 \mathrm{~h}$, but did not survive for $24 \mathrm{~h}$, were also defined as having required MT in order to reduce survivor bias [7,10]. After the source of bleeding had been identified and hemorrhaging had been controlled via surgical or catheter intervention, the necessity of MT was determined based on clinical judgments. The patients who required MT received transfusions of RCC, fresh frozen plasma (FFP), and platelet concentrates (PC) at a 1:1:1 ratio. RCC, FFP, and PC were administered to maintain a hemoglobin level of $\geq 7.0 \mathrm{~g} / \mathrm{dl}$, an international normalized ratio (INR) of $\leq 1.5$, a fibrinogen level of $\geq 200 \mathrm{mg} / \mathrm{dl}$, and a platelet count of $\geq 5 \times 10^{4} / \mu \mathrm{l}$ on repeated laboratory examinations.

\section{Data collection}

The following data were recorded: age; sex; the mechanism of injury (blunt or penetrating); heart rate; systolic blood pressure; shock index (SI, defined as the ratio of heart rate to systolic blood pressure); base excess (BE); serum lactate level; hemoglobin level on arrival; the results of focused assessments of sonography for trauma (FAST); the presence or absence of pericardial effusion, intrathoracic fluid, or intraabdominal fluid; the injury severity score (ISS); the total amount of transfused products delivered within $24 \mathrm{~h}$; and the outcome at hospital discharge.

\section{Statistical analysis}

Categorical variables are shown as frequencies or percentages, whereas continuous variables are presented as mean and standard deviation (SD) values or median and interquartile range values depending on their distributions. Categorical variables were compared using Fisher's exact probability test. Student's $t$ test was used to assess continuous variables with normal distributions, and the Mann-Whitney $U$-test was used to evaluate variables with non-normal distributions. We used multiple logistic

Table 1 Baseline characteristics of the MT and non-MT groups

\begin{tabular}{|c|c|c|c|}
\hline & MT group & non-MT group & $P$ value \\
\hline Age (year) & $53 \pm 20$ & $49 \pm 20$ & 0.22 \\
\hline Males, n (\%) & $24(73)$ & $154(68)$ & 0.60 \\
\hline Blunt mechanism, $n(\%)$ & $30(91)$ & $228(95)$ & 0.25 \\
\hline $\mathrm{HR}$ (beats/min), mean $\pm \mathrm{SD}$ & $106 \pm 31$ & $87 \pm 16$ & $<0.001$ \\
\hline $\mathrm{SBP}(\mathrm{mmHg})$, mean $\pm \mathrm{SD}$ & $99 \pm 36$ & $136 \pm 27$ & $<0.001$ \\
\hline Shock index, median (IQR) & $1.04(0.79,1.50)$ & $0.63(0.52,0.77)$ & $<0.001$ \\
\hline Body surface temperature $\left({ }^{\circ} \mathrm{C}\right)$, median $(\mathrm{IQR})$ & $36.1(35.7,36.6)$ & $36.6(36.1,36.9)$ & 0.002 \\
\hline $\mathrm{BE}(\mathrm{mmol} / \mathrm{l})$, median $(\mathrm{IQR})$ & $-4.8(-8.2,-2.4)$ & $-0.3(-2.2,1.0)$ & $<0.001$ \\
\hline Lactate $(\mathrm{mmol} / \mathrm{l})$, median (IQR) & $3.7(2.8,5.7)$ & $2.0(1.3,2.7)$ & $<0.001$ \\
\hline Hemoglobin $(\mathrm{g} / \mathrm{dl})$, median (IQR) & $12.4(10.7,13.4)$ & $13.8(12.3,15.0)$ & $<0.001$ \\
\hline Platelet count $(\times 104 / \mu \mid)$, median (IQR) & $19.3(15.6,22.3)$ & $21.5(18.1,25.4)$ & 0.013 \\
\hline Fibrinogen (mg/dl), median (IQR) & $247(192,312)$ & $324(272,376)$ & $<0.001$ \\
\hline INR, median (IQR) & $1.02(0.95,1.37)$ & $0.92(0.88,0.98)$ & $<0.001$ \\
\hline D-dimer $(\mu \mathrm{g} / \mathrm{ml})$, median $(\mathrm{IQR})$ & $32.0(12.6,101.8)$ & $4.6(0.9,19.1)$ & $<0.001$ \\
\hline Positive FAST result, $n$ (\%) & $20(61)$ & $16(7)$ & $<0.001$ \\
\hline Pelvic fracture, $n(\%)$ & $14(42)$ & $14(6)$ & $<0.001$ \\
\hline ISS, median (IQR) & $41(30,45)$ & $9(2,17)$ & $<0.001$ \\
\hline RCC (units), median (IQR) & $20(13,31)$ & $0(0,0)$ & $<0.001$ \\
\hline FFP (units), median (IQR) & $12(8,22)$ & $0(0,0)$ & $<0.001$ \\
\hline PC (units), median (IQR) & $20(0,45)$ & $0(0,0)$ & $<0.001$ \\
\hline Mortality, $n(\%)$ & $9(27)$ & $3(1)$ & $<0.001$ \\
\hline
\end{tabular}

Shock index: heart rate/systolic blood pressure. $M T$ massive transfusion, $H R$ heart rate, $S D$ standard deviation, $S B P$ systolic blood pressure, IQR interquartile range, $B E$ base excess, INR international normalized ratio, FAST focused assessment of sonography for trauma, ISS injury severity score, RCC red cell concentrate, FFP fresh frozen plasma, $P C$ platelet concentrate. 
regression analysis to identify independent predictors of the necessity of MT. The ability of the resultant model to predict the necessity of MT was estimated based on the area under the receiver operating characteristic curve (AUROC). $P$ values of $<0.05$ were considered to be statistically significant. All analyses were performed using the software SPSS for Windows (release 19.0).

\section{Results}

A total of 259 trauma patients were included in this study (males: 178, 69\%). Their mean age was $49 \pm 20$, and their median ISS was 14.4. Thirty-three (13\%) patients required $\mathrm{MT}$, and the overall mortality rate was $4.6 \%(n=12)$. Of the 33 patients who required MT, two died within $24 \mathrm{~h}$ and did not actually receive MT (one due to hemorrhagic shock and the other one due to a severe traumatic brain injury complicated with hemorrhagic shock). The causes of death in the seven patients who died after receiving MT were as follows: catastrophic brain injury (two patients), severe traumatic brain injury complicated with hemorrhagic shock (four patients), and exsanguination (one patient). The baseline characteristics of the MT and non-MT groups are shown in Table 1 . In the MT group, the median total amounts of RCC, FFP, and PC administered were 20, 12, and 20 units, respectively. Among these predictors of the necessity of MT, we subjected a high shock index, a reduced $\mathrm{BE}$, lower level of hemoglobin, and a positive FAST result to multivariate analysis, as clinically important, these parameters can be assessed within a few minutes of arrival at the ED and so might be useful for establishing an easy and simplified screening method for determining the necessity of MT. Multivariate analysis revealed that a high shock index, a reduced $\mathrm{BE}$, and a positive FAST result were predictors of MT being required (Table 2). Thus, we created a new simplified screening model based on these three predictors, i.e., a shock index of $\geq 1$, a $\mathrm{BE}$ of $\leq-3 \mathrm{mmol} / \mathrm{l}$, and a positive FAST result. In our scoring system, one point was awarded for each of these components, and hence, the total score ranged from 0 to 3 . Shock index and $\mathrm{BE}$ cut-off points were determined based on AUROC values, as shown in Table 3.

Table 2 Multivariate analysis of predictors of massive transfusion

\begin{tabular}{lll}
\hline Variables & Odds ratio $(\mathbf{9 5} \% \mathrm{Cl})$ & $\boldsymbol{P}$ value \\
\hline Shock index & $259.81(17.85-3782.41)$ & $<0.001$ \\
BE & $0.72(0.58-0.89)$ & 0.002 \\
Hemoglobin & $0.96(0.76-1.22)$ & 0.743 \\
Positive FAST result & $7.28(2.24-23.61)$ & 0.001 \\
\hline
\end{tabular}

Cl confidence interval, $B E$ base excess, FAST focused assessment of sonography for trauma.
Table 3 AUROC values obtained for our new screening method using different shock index and BE cut-off points

\begin{tabular}{lllll}
\hline Shock index & BE & FAST & AUROC & 95\% Cl \\
\hline$\geqq 1$ & $\leqq-2.0$ & positive & 0.919 & $0.869-0.968$ \\
$\geqq 1$ & $\leqq-3.0$ & positive & 0.934 & $0.891-0.978$ \\
$\geqq 1$ & $\leqq-4.0$ & positive & 0.928 & $0.875-0.981$ \\
$\geqq 0.8$ & $\leqq-3.0$ & positive & 0.909 & $0.858-0.960$ \\
$\geqq 1.5$ & $\leqq-3.0$ & positive & 0.895 & $0.831-0.959$ \\
\hline
\end{tabular}

$A U R O C$ area under the receiver operating characteristic curve, $B E$ base excess, FAST focused assessment of sonography for trauma, $\mathrm{Cl}$ confidence interval.

\section{Discussion}

Exsanguination is the most frequent acute cause of death (51\%) in trauma patients [1], and persistent hemorrhagic shock that requires MT eventually results in multiple organ failure [11]. DCR is an integrated approach used to treat severely injured trauma patients. In this method, damage control surgery, which is a surgical technique that focuses on controlling hemorrhaging and contamination, is first performed. Then, blood pressure is maintained below approximately $90 \mathrm{mmHg}$ to prevent further bleeding, and multiple blood products and limited crystalloid fluid resuscitation are employed to treat and prevent coagulopathy. DCR must be initiated immediately after arrival in the ED and is continued in both the operating room and the intensive care unit $[3,10,12,13]$. As for MT, several studies have proposed predictive models for identifying trauma patients who require MT [6-9]. These models are each composed of several variables, e.g., basic data such as age and the mechanism of injury; vital signs such as heart rate and systolic blood pressure; laboratory data such as $\mathrm{BE}$, serum lactate, hemoglobin, and INR values; and diagnostic imaging findings such as FAST results and parameters derived from X-rays of the pelvis. Among these models, the assessment of blood consumption (ABC) score, which is based on the mechanism of injury, heart

Table 4 The ABC scoring system and our new screening method

\begin{tabular}{ll}
\hline & Points \\
\hline ABC score & 1 \\
Penetrating mechanism & 1 \\
SBP of $\leq 90 \mathrm{mmHg}$ & 1 \\
HR of $\geq 120 \mathrm{bpm}$ & 1 \\
Positive FAST result & \\
Our new screening method & 1 \\
Shock index of $\geq 1$ & 1 \\
BE of $\leq-3.0$ mmol/L & 1 \\
Positive FAST result & \\
\hline
\end{tabular}

$A B C$ score assessment of blood consumption score, FAST focused assessment of sonography for trauma, SBP systolic blood pressure. 
Table 5 Sensitivity and specificity values and the percentage of correctly classified cases obtained with the $A B C$ scoring system and our new screening method (Table 4)

\begin{tabular}{lllll}
\hline & $\begin{array}{l}\text { Cut-off } \\
\text { point }\end{array}$ & $\begin{array}{l}\text { Sensitivity } \\
\text { (\%) }\end{array}$ & $\begin{array}{l}\text { Specificity } \\
\text { (\%) }\end{array}$ & $\begin{array}{l}\text { Correctly } \\
\text { classified (\%) }\end{array}$ \\
\hline ABC score in original & $\geqq 0$ & 100 & 0 & 13 \\
study & $\geqq 1$ & 95 & 56 & 61 \\
& $\geqq 2$ & 75 & 86 & 84 \\
& $\geqq 3$ & 25 & 97 & 87 \\
& $\geqq 4$ & 6 & 100 & 88 \\
ABC scores obtained & $\geqq 0$ & 100 & 0 & 13 \\
using our center's data & $\geqq 1$ & 85 & 85 & 46 \\
& $\geqq 2$ & 48 & 98 & 80 \\
& $\geqq 3$ & 19 & 100 & 100 \\
& $\geqq 4$ & 0 & 100 & - \\
Our new screening & $\geqq 0$ & 100 & 0 & 13 \\
method & $\geqq 1$ & 97 & 81 & 43 \\
& $\geqq 2$ & 61 & 93 & 56 \\
& $\geqq 3$ & 36 & 98 & 69 \\
\hline
\end{tabular}

$A B C$ score assessment of blood consumption score, $B E$ base excess, FAST focused assessment of sonography for trauma, SBP systolic blood pressure, $H R$ heart rate.

rate, systolic blood pressure, and FAST results, was developed as a simplified model for predicting the necessity of MT early [7]. This score of $\geq 2$ exhibits a sensitivity value of $75 \%$, a specificity value of $86 \%$, and a positive predictive value of $84 \%$. However, when the $\mathrm{ABC}$ scoring system was applied to our data, an $\mathrm{ABC}$ score of $\geq 2$ only exhibited a sensitivity value of $48 \%$ (Table 4 ). It is considered that the $\mathrm{ABC}$ score exhibits low sensitivity in Japan because blunt mechanisms account for the majority of injuries experienced in this country. Secondary, there were a total of 17 false negative patients using $A B C$ score of $\geq 2$. Of them, eight patients were complicated with severe traumatic brain injury. These patients have not necessarily presented with hypotension and tachycardia on arrival. These factors are associated with low sensitivity value of $A B C$ score. As for our new screening model, $97 \%$ of patients who required MT met at least one of the abovementioned three conditions (Table 5). Figure 1 shows the AUROC of the $\mathrm{ABC}$ score and our new screening model. Our model exhibited a strong ability to predict the necessity of MT. In the presence of at least one of the three conditions, our model displayed high sensitivity and specificity for predicting the necessity of MT; however, its positive predictive value was only $43 \%$ (Table 5). Our screening method, which is based on the presence of a high shock index, a low BE value, and/or a positive FAST result, displayed high levels of accuracy during ROC analysis. In addition, this screening method can be completed within minutes of the patient's arrival in the ED, which would allow MT protocols to be initiated quickly. Although our method exhibits high sensitivity and specificity, its positive predictive value is very low; thus, it might not be useful for diagnostic purposes.

This was a single-center retrospective study involving a small population, and several other important limitations also exist. For example, our results might not be applicable to institutions that use different MT protocols or trauma systems or to different patient populations. Although our patients were managed with the aim of attaining a high FFP to RCC transfusion ratio, the actual ratio achieved was relatively low (0.6:1), which suggests that some patients might not have actually required MT. Several studies have reported that a high FFP to RCC ratio improves the outcomes of severely injured trauma patients, but we have to take survivor bias into consideration $[14,15]$. Another point is that some patients could have avoided MT if earlier or alternative methods have been employed to control their bleeding. Further investigation is necessary to determine whether our screening

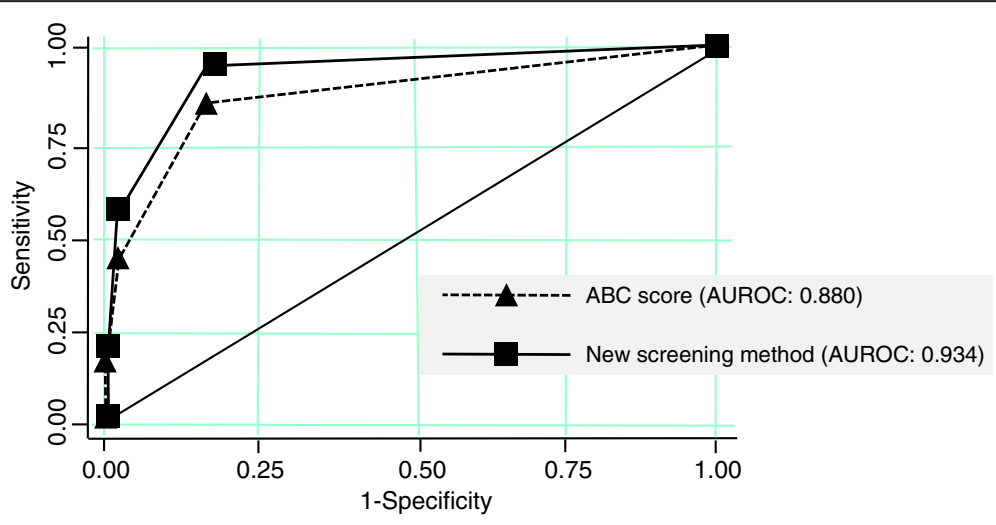

AUROC: area under the receiver operating characteristic curve, $A B C$ score: assessment of blood consumption score

Figure 1 AUROC values for the $A B C$ scoring system and our new screening method. 
system facilitates the early initiation of MT protocols and the rapid control of hemorrhaging.

\section{Conclusions}

Screening based on a combination of a high shock index, a low BE value, and/or a positive FAST result is an easy and useful way of predicting the necessity of MT in trauma patients; however, this approach might not be useful for diagnostic purposes.

\section{Abbreviations}

MT: Massive transfusion; FAST: positive focused assessment of sonography for trauma; DCR: damage control resuscitation; ED: emergency department; RCC: red cell concentrations; FFP: fresh frozen plasma; PC: platelet concentrates; INR: international normalized ratio; SI: shock index; BE: base excess; ISS: injury Severity Score; AUROC: area under the receiver operating characteristic curve; SD: standard deviations; IQR: interquartile ranges; $A B C$ : assessment of blood consumption.

\section{Competing interests}

The authors declare that they have no competing interests.

\section{Authors' contributions}

TY planed and conducted the study. TY, Al, TH, KT, NS, and HY collected the data. KS, TU, and SI helped to coordinate the study and draft the manuscript. YU supervised and edited the study. All authors read and approved the final manuscript.

Received: 20 June 2014 Accepted: 29 August 2014

Published online: 10 September 2014

\section{References}

1. Sauaia A, Moore FA, Moore EE, Moser KS, Brennan R, Read RA, Pons PT: Epidemiology of trauma deaths: a reassessment. J Trauma 1995, 38:185-193.

2. Jansen JO, Thomas R, Loudon MA, Brooks A: Damage control resuscitation for patients with major trauma. BMJ 2009, 5(338):b1778.

3. Fraga GP, Bansal V, Coimbra R: Transfusion of blood products in trauma: an update. J Emerg Med 2010, 39:253-260.

4. Brockamp T, Nienaber U, Mutschler M, Wafaisade A, Peiniger S, Lefering R, Bouillon B, Maegele M: TraumaRegister DGU: predicting on-going hemorrhage and transfusion requirement after severe trauma: a validation of six scoring systems and algorithms on the Trauma Register DGU ${ }^{\bullet}$. Crit Care 2012, 16:R129.

5. Gruen RL, Brohi K, Schreiber M, Balogh ZJ, Pitt V, Narayan M, Maier RV: Haemorrhage control in severely injured patients. Lancet 2012, 380:1099-1108

6. Yücel N, Lefering R, Maegele M, Vorweg M, Tjardes T, Ruchholtz S, Neugebauer EAM, Wappler F, Bouillon B, Rixen D: Trauma Associated Severe Hemorrhage (TASH)-Score: probability of mass transfusion as surrogate for life threatening hemorrhage after multiple trauma. J Trauma 2006, 60:1228-1236. discussion 1236-1237.

7. Nunez TC, Voskresensky IV, Dossett L, Shinall R, Dutton WD, Cotton B: Early prediction of massive transfusion in trauma: simple as $A B C$ (assessment of blood consumption)? J Trauma 2009, 66:346-352.

8. Rainer TH, Ho AM-H, Yeung JHH, Cheung NK, Wong RSM, Tang N, Ng SK, Wong GKC, Lai PBS, Graham CA: Early risk stratification of patients with major trauma requiring massive blood transfusion. Resuscitation 2011, 82:724-729.

9. Schreiber M, Perkins J, Kiraly L, Underwood S, Wade C, Holcomb JB: Early predictors of massive transfusion in combat casualties. J Am Coll Surg 2007, 205:541-545.

10. Kutcher ME, Kornblith LZ, Narayan R, Curd V, Daley AT, Redick BJ, Nelson $M F$, Fiebig EW, Cohen MJ: A paradigm shift in trauma resuscitation: evaluation of evolving massive transfusion practices. JAMA Surg 2013, 148:834-840.

11. Sauaia A, Moore FA, Moore EE, Haenel JB, Read RA, Lezotte DC: Early predictors of postinjury multiple organ failure. Arch Surg 1994, 129:39-45.

12. Holcomb JB, Jenkins $D$, Rhee P, Johannigman J, Mahoney P, Mehta S, Cox ED, Gehrke MJ, Beilman GJ, Schreiber M, Flaherty SF, Grathwohl KW, Spinella
PC, Perkins JG, Beekley AC, MCMullin NR, Park MS, Gonzalez EA, Wade CE, Dubick MA, Schwab CW, Moore FA, Champion HR, Hoyt DB, Hess JR: Damage control resuscitation: directly addressing the early coagulopathy of trauma. J Trauma 2007, 62:307-310.

13. Cohen MJ: Towards hemostatic resuscitation: the changing understanding of acute traumatic biology, massive bleeding, and damage-control resuscitation. Surg Clin North Am 2012, 92:877-891.

14. Zink KA, Sambasivan CN, Holcomb JB, Chisholm G, Schreiber MA: A high ratio of plasma and platelets to packed red blood cells in the first 6 hours of massive transfusion improves outcomes in a large multicenter study. Am J Surg 2009, 197:565-570.

15. Ho AM, Dion PW, Yeung JH, Holcomb JB, Critchley LA, Ng CS, Karmakar MK, Cheung CW, Rainer TH: Prevalence of survivor bias in observational studies on fresh frozen plasma: erythrocyte ratios in trauma requiring massive transfusion. Anesthesiology 2012, 116:716-728.

doi:10.1186/s40560-014-0054-3

Cite this article as: Yumoto et al:: Immediate screening method for predicting the necessity of massive transfusions in trauma patients: a retrospective single-center study. Journal of Intensive Care 2014 2:54.

\section{Submit your next manuscript to BioMed Central and take full advantage of:}

- Convenient online submission

- Thorough peer review

- No space constraints or color figure charges

- Immediate publication on acceptance

- Inclusion in PubMed, CAS, Scopus and Google Scholar

- Research which is freely available for redistribution 